\title{
Caracterización del FinANCIAMIENTO EN MICRO Y PEQUEÑas EMPRESAS, RUBRO CHIFAS DE SANTA y COISHCO, 2013
}

\section{ChARACTERIZATION OF FINANCING IN THE SERVICE SECTOR MYPES - ITEM CHIFAS CITIES OF SANTA AND COISHCO, 2013}

\author{
Franchestka S. Romero Olaza* \\ Reinerio Centurión Medina**
}

\begin{abstract}
RESUMEN
I a presente investigación tuvo por objetivo determinar las principales características Ldel financiamiento en las mypes del sector servicio - rubro chifas en las ciudades de Santa y Coishco, 2013. La investigación fue de tipo cuantitativo, nivel descriptivo y de diseño no experimental/transversal. Se utilizó una población muestral de 5 mypes, a quienes se les aplicó un cuestionario estructurado de 12 preguntas con la técnica de la encuesta obteniéndose los siguientes resultados: en referencia al representante legal de las mypes, el $100 \%$ tiene edades entre los 26-60 años. El $80 \%$ tiene ambos mitad por mitad estudios primarios y secundarios, y solo el $20 \%$ tiene estudios superiores no universitarios. En relación con las mypes, el 80 \% tiene de funcionamiento mitad por mitad de 1, 3 y 5 años a más y solo el $20 \%$ tiene de funcionamiento de 3 años- 5 años. El 100 \% estableció la creación de su mype por una oportunidad en el mercado. Referente al financiamiento: el $80 \%$ tiene financiamiento propio y el otro $20 \%$ tiene financiamiento de terceros. El $60 \%$ al no solicitar crédito de ninguna entidad, solo el 40 $\%$ obtuvieron crédito mitad por mitad de una entidad bancaria y no bancaria. El 100 $\%$ considera que las entidades bancarias les otorgan mayores facilidades. El $80 \%$ al no solicitar crédito de ninguna entidad, solo el 20 \% utilizó el crédito obtenido para el mejoramiento y/o ampliación de su local.
\end{abstract}

Palabras clave: mypes, financiamiento.

\footnotetext{
* Egresado de la Escuela Profesional de Administración-Uladech Católica.

** Magíster en Administración. Docente tutor, investigador de la Escuela Profesional de Administración-ULADECH Católica.
} 


\begin{abstract}
This research aimed to determine the main characteristics of financing MSE service industry - chifas category in the cities of Santa and Coishco, 2013. The research was quantitative, descriptive level and not experimental / cross-sectional design was used a sample population of 5 MSE who were administered a structured 12-question questionnaire through the survey technique with the following results: Regarding the legal representative of the MSE: $100 \%$ is aged between 26 years - 60 years. $80 \%$ have both half and half primary and secondary education, and only $20 \%$ are nonuniversity higher education. Regarding MSE: $80 \%$ have running half and half than 1 year- 3 years and 5 years - more and only $20 \%$ have running 3 years- 5 years. $100 \%$ established the creation of the MSE for an opportunity in the market. Concerning the financing: $80 \%$ have their own funding and the other $20 \%$ have third party financing. The $60 \%$ credit by not requesting any entity, only $40 \%$ half and half obtained credit from a bank and nonbank. $100 \%$ believe that banks give them greater facilities. The 80 $\%$ credit by not requesting any entity, only $20 \%$ used credit earned for the improvement and / or expansión of your local .
\end{abstract}

Keywords: MSE, financing.

\title{
INTRODUCCIÓN
}

Las micro y pequeñas empresas (mypes) constituyen uno de los principales motores de la actividad económica, los cuales han incrementado su participación en los diferentes sectores productivos de la economía en los últimos años, incitando al crecimiento y desarrollo del país. Es por ello que las instituciones bancarias, hoy en día, han volcado la mirada a estas pequeñas unidades económicas y las ven rentables en cuanto al financiamiento de crédito, a pesar del alto riesgo que conlleva dicha acción (Bustamante, s. f.).

El mundo que nos rodea avanza a una velocidad acelerada, la cual puede notarse en los ámbitos de economía, cultura y entre otros. Tenemos el caso de Europa, donde las pequeñas o medianas empresas (pyme) pueden beneficiarse de la financiación de la Unión Europea (UE) a través de subvenciones, préstamos y, en algunos casos, garantías. El apoyo puede ser directo (subvenciones de la UE) o a través de programas de ámbito nacional, cuentan también con una serie de medidas de ayuda no financiera en forma de programas y servicios de apoyo empresarial prestados por la Red Empresarial Europea o la Ventanilla sobre Derechos de Propiedad Intelectual (García, 2013). Se observa también que las pymes españolas requieren especial atención por dos motivos: 1) porque constituyen el 99,9 \% del total de las empresas; y 2) porque son altamente dependientes de la financiación bancaria; en concreto, el crédito bancario representa el $40 \%$ de su financiación ajena por eso cualquier 
estrategia de salida de la crisis exige mejorar las condiciones de la financiación de las pymes (Ferraro, 2011).

En el caso de América Latina, las mypes ya han adquirido un perfil identificable por su formalidad, ventas, gastos, insumos, formas de pago, instrumentos financieros usados, acceso al crédito y perspectivas de futuro. En Panamá, uno de los grandes problemas de las mypes deriva de su falta de acceso al financiamiento, precisamente la Autoridad de las Pequeñas y Medianas Empresas ha venido ejecutando diversas iniciativas como el Programa Fideicomiso para el Financiamiento de la Competitividad y Productividad (Findec) que constituye un fondo que facilita que los bancos comerciales provean fondos para que las empresas puedan invertir en capacidad productiva y capital de trabajo (Tam, 2010). En Brasil, el Sebrae se focaliza en la provisión de soluciones para facilitar el acceso al financiamiento para las empresas de menor tamaño; estas incluyen la información sobre los programas y líneas de crédito existentes, consultorías para realizar diagnósticos financieros y el armado de los proyectos, etc. Además, pueden participar en el Fondo de Garantías para las Micro y Pequeñas Empresas (Fampe) e impulsa la creación de sociedades de garantías con el objetivo de ofrecer alternativas para la cobertura de los créditos de las empresas (Morais, 2009).

Ya en el Perú, se observa que el número de micro y pequeñas empresas es del $98.35 \%$ del total de empresas, pero como se puede apreciar, la realidad generadora de estas micro y pequeñas empresas es limitada, porque no están en competencia internacional. Ante estos problemas el Gobierno ha creado un programa especial de apoyo financiero para las mypes, el cual contará inicialmente con 200 millones de recursos en el Banco de la Nación, pero esto no es suficiente porque solo van a favorecer a 100 mil micro y pequeñas empresas del Perú (Alaya, 2009). El Fondo de Apoyo para la Micro, Pequeña y Mediana Empresa (Fondo PYME) es un instrumento que busca apoyar particularmente a las empresas de menor tamaño y a los emprendedores, con el propósito de promover el desarrollo económico nacional y estatal, a través del otorgamiento de apoyos de carácter temporal a programas y proyectos que fomenten la creación, desarrollo, viabilidad, productividad, competitividad y sustentabilidad de las micro, pequeñas y medianas empresas (Torres, 2013). Como se puede observar el gran problema que tienen las mypes, el mismo que impide su formalización, es el financiamiento, pues el mismo se hace a través de los bancos privados, siendo demasiado onerosos en el cobro de los intereses, donde consigue los créditos, vía Cofide, a 8 \% o $10 \%$ de interés y les presta a las mypes cobrándoles intereses que se elevan hasta el 30 \% (Latínez, 2013).

Actualmente, las mypes de la región Áncash presentan una deficiente organización a nivel empresarial que involucra aspectos económicos, financieros y administrativos, que impide el desarrollo de experiencias asociativas de tipo gremial y empresarial.

En la ciudad de Santa donde se desarrolló la presente investigación se centra en los negocios conocidos como mypes dedicadas al rubro de chifas, de las cuales se desconoce si estas tienen o no acceso al financiamiento. Y, si es así, cuánto de interés pagan por el crédito y mediante qué sistema financiero obtuvieron el crédito; por todo ello, el enunciado del problema nos induce a plantear la siguiente pregunta de investigación: ¿Cuáles son las principales características del financiamiento de las mypes del sector servicio - rubro chifas en las ciudades de Santa y Coishco, 2013? 
Para dar respuesta al problema, se ha planteado el objetivo general de describir las principales características del financiamiento en las mypes del sector serviciorubro chifas en las ciudades de Santa y Coishco, 2013. Para poder conseguir el objetivo general, nos hemos planteado los siguientes objetivos específicos que dan sustento a esta problemática los cuales son determinar las características en las mypes del sector servicio -rubro chifas en las ciudades de Santa y Coishco; determinar las características de los representantes legales que dirigen en las mypes del sector servicio - rubro chifas en las ciudades de Santa y Coishco, 2013; determinar las características del financiamiento en las mypes del sector servicio - rubro chifas en las ciudades de Santa y Coishco, 2013.

Finalmente, la investigación permitió determinar las principales características del financiamiento en las mypes en estudio, a través de información precisa, clara y actualizada, lo cual tendrá como resultado que los estudiantes puedan acceder a ella de manera fácil y que les será de gran utilidad. Los beneficios que se obtendrán con esta investigación será la generación de un mayor número de empleos y el de nuevas organizaciones que se entusiasmarán a conformar su empresa. Por último, la investigación contribuirá a que se puedan realizar nuevos estudios. Este significará el punto de partida para muchos investigadores que desean profundizar el tema en diversos ámbitos geográficos del país.

\section{MATERIALES Y MÉTODOS}

Para la elaboración del presente trabajo de investigación se utilizó el diseño no experimental-transversal-descriptivo-cuantitativo. Fue no experimental porque se realizó sin manipular deliberadamente las variables; es decir, se observó el fenómeno tal como se encuentra dentro de su contexto, conforme a la realidad, sin sufrir modificaciones.

Fue transversal porque el estudio se realizó en un espacio de tiempo determinado, donde se tuvo un inicio y un fin. Fue descriptivo porque solo se describieron las características más relevantes de las mypes, representantes y la variable en estudio.

Fuecuantitativaporquetuvounaestructura conla cualsepudomedir,yaque seutilizaron instrumentos de evaluación. Se utilizó una población muestral porque se tomó a toda la población de las mypes del sector servicio - rubro chifas de la ciudad de Santa, 2013, para la realización del estudio. La técnica que se utilizó, para recolectar información fue la encuesta, porque es una técnica destinada a obtener datos de varias personas cuyas opiniones impersonales interesan al investigador.

El instrumento que se utilizó para registrar la información fue el cuestionario, el cual es un instrumento que consta de un conjunto de preguntas, preparadas cuidadosamente, sobre los hechos y aspectos que interesan en una investigación.

El presente estudio de investigación titulado "Caracterización del financiamiento en las mypes del sector servicio - rubro chifas de la ciudad de santa, 2013". Para la realización del estudio.

Se encuentra en la etapa de trabajo de campo, el cual consiste en recolectar toda la información necesaria para tener un sustento con respecto al trabajo de investigación. Para obtener toda la información necesaria se tuvo en cuenta una población muestral de 2 mypes del sector servicio - rubro chifas de las ciudades de Santa y Coishco, 2013. 
Teniendo el cuestionario ya estructurado con 12 preguntas, se procedió a aplicar dicho instrumento a través de la técnica de la encuesta a una población muestral dirigida de 5 mypes, obteniéndose los siguientes resultados detallados a continuación:

\section{RESULTADOS}

Cuadro 1. Respecto a los representantes de la mype.

\begin{tabular}{ll}
\hline \multicolumn{1}{c}{ ÍTEM } & \multicolumn{1}{c}{ RESULTADOS } \\
\hline Edad & El $100 \%$ tiene entre los 26-60 años. \\
Sexo & El $80 \%$ es del sexo masculino y el otro $20 \%$ es del sexo femenino. \\
Grado de instrucción & El $40 \%$ tiene el grado de instrucción primaria, $40 \%$ tiene hasta la \\
& secundaria y solo el $20 \%$ superior no universitaria.
\end{tabular}

Cuadro 2. Respecto a las características de la mype.

\begin{tabular}{ll}
\hline \multicolumn{1}{c}{ ÍTEM } & \multicolumn{1}{c}{ RESULTADOS } \\
\hline $\begin{array}{l}\text { Tiempo de funcionamiento de su } \\
\text { empresa }\end{array}$ & $\begin{array}{l}\text { El } 40 \% \text { tiene de funcionamiento de } 1-3 \text { años, } 40 \text { \% tiene de } 5 \text { años a } \\
\text { más y el otro } 20 \% \text { tiene de 3-5 años. }\end{array}$ \\
$\begin{array}{ll}\text { Motivo de la creación de las mypes El } 100 \% \text { estableció que la creación de su mype por una oportunidad } \\
\text { en el mercado. }\end{array}$
\end{tabular}

Fuente: Elaboración propia

Cuadro 3. Respecto al financiamiento de la mype.

\begin{tabular}{|c|c|}
\hline ÍTEM & RESULTADOS \\
\hline $\begin{array}{l}\text { Financiamiento de su actividad } \\
\text { productiva }\end{array}$ & $\begin{array}{l}\text { El } 80 \% \text { tiene financiamiento propio y el otro } 20 \% \text { tiene } \\
\text { financiamiento de tercero. }\end{array}$ \\
\hline $\begin{array}{l}\text { Tipos de entidades financieras que ha } \\
\text { obtenido crédito }\end{array}$ & $\begin{array}{l}\text { El } 80 \% \text { al no solicitar crédito de ninguna entidad, solo el } \\
20 \% \text { ha obtenido crédito de una entidad bancaria y el otro } \\
20 \% \text { ha obtenido crédito de una entidad no bancaria. }\end{array}$ \\
\hline Requisito que le pidieron por el crédito & $\begin{array}{l}\text { El } 80 \% \text { al no solicitar crédito de ninguna entidad, solo al } \\
20 \% \text { le pidieron de requisito alguna hipoteca. }\end{array}$ \\
\hline $\begin{array}{l}\text { Entidades financieras que otorgan } \\
\text { mayores facilidades para la } \\
\text { obtención del crédito }\end{array}$ & $\begin{array}{l}\text { Al } 100 \% \text { le otorgan mayores facilidades las entidades } \\
\text { bancarias. }\end{array}$ \\
\hline Monto aproximado que solicitó & $\begin{array}{l}\text { El } 80 \% \text { al no solicitar crédito de ninguna entidad, solo al } \\
20 \% \text { le dieron un monto aproximado de 10,001-más. }\end{array}$ \\
\hline Utilización del crédito obtenido & $\begin{array}{l}\text { El } 80 \text { \% al no solicitar crédito de ninguna entidad, solo el } \\
20 \% \text { utilizó el crédito obtenido para el mejoramiento y/o } \\
\text { ampliación de su local. }\end{array}$ \\
\hline Tasa de interés por el crédito & $\begin{array}{l}\text { El } 80 \% \text { al no solicitar crédito de ninguna entidad, solo el } \\
20 \% \text { pagó una tasa de interés del 11-20\% por el crédito } \\
\text { obtenido. }\end{array}$ \\
\hline
\end{tabular}

Fuente: elaboración propia 


\section{DISCUSIÓN}

\section{Referente a los representantes legales de las mypes}

1. En el cuadro 1: El $100 \%$ de los representantes tiene edades entre los 26-60 años. Esta coincide con los resultados de Silva (2013), donde la edad oscila entre 26-60 años, al igual que Vásquez (2008), donde la edad promedio fue de 42 años. Esto demuestra que actualmente las mypes están dirigidas por personas adultas, ya que cuentan con una mayor experiencia y que se desenvuelven mejor en el ámbito en que se están desarrollando.

2. En el cuadro 2: El $80 \%$ de los representantes son del sexo masculino y el otro $20 \%$ son del sexo femenino, al igual que Vásquez (2008), donde el 54 \% fueron del sexo masculino. Esto demuestra que actualmente las mypes están dirigidas de una manera equiparada por ambos sexos, donde ya no hay diferencias.

3. En el cuadro 3: El $80 \%$, ambos mitad por mitad, tienen estudios primarios y secundarios, y solo el $20 \%$ tiene estudios superior no universitario, esto coincide con los resultados de Reyna (2008), el 66.7 \% tienen estudios secundarios. Esto demuestra que la mayoría de los representantes de las mypes tienen estudios secundarios y que de esa manera ellos comenzaron a incursionar en este tipo de negocio.

\section{Referente a las mypes}

4. En el cuadro 4: El $80 \%$ ambos mitad por mitad tiene de funcionamiento de 1-3 años y de 5 años a más y solo el $20 \%$ tiene de funcionamiento de 3-5 años, a diferencia de los resultados de Vásquez (2008) tienen más de 3 años de antigüedad. Esto demuestra que actualmente las mypes tienen 1-3 años y el de 5 años a más las cuales han sabido mantenerse en el mercado durante este tiempo a pesar de las dificultades que se le haya podido presentar.

5. En el cuadro 5: El 100 \% estableció la creación de su MYPE fue por una oportunidad en el mercado. Esto nos demuestra que actualmente la creación de las mypes está orientada por una oportunidad en el mercado, donde los representantes han sabido hacia donde enfocarse, es de ahí los logros que han ido obteniendo.

\section{Referente al financiamiento}

6. En el cuadro 6: El $80 \%$ tiene financiamiento propio y el otro $20 \%$ tiene financiamiento de terceros. Esto demuestra que las mypes han sido financiadas por financiamiento propio, lo cual les ha ayudado a que se puedan posicionar en el mercado al que están enfocados. 
7. En el cuadro 7: El $60 \%$ al no solicitar crédito de ninguna entidad, solo el $40 \%$ ambos mitad por mitad obtuvieron crédito de una entidad bancaria y no bancaria, a diferencia de los resultados de (Silva, 2013), donde en el año 2008 el $42.86 \%$ de los empresarios encuestados recibieron créditos y en el año 2009 nadie recibía crédito. Esto demuestra que actualmente la mayoría de los representantes de las mypes no han obtenido crédito de ninguna entidad, el motivo es porque no quieren involucrarse en este tipo de proceso, ya que tienen una concepción errónea y piensan que son varios los requisitos para su solicitud el crédito o por la alta tasas de interés que cobran.

8. En el cuadro 8: El $80 \%$ al no solicitar crédito de ninguna entidad, solo al $20 \%$ le pidieron de requisito alguna hipoteca. Esto demuestra que actualmente la gran mayoría de los representantes de las mypes no le han pedido ningún requisito, ya que estos no han accedido a la obtención de crédito en una entidad bancaria o no bancaria.

9. En el cuadro 9: El $100 \%$ considera que las entidades bancarias le otorgan mayores facilidades. Esto demuestra que actualmente a los representantes de las mypes les brindan mayores facilidades las entidades bancarias, donde ya tienen establecidos los procedimientos a seguir y las indicaciones a dar a los que solicitan el crédito de manera clara y concreta.

10. En el cuadro 10: El $80 \%$ al no solicitar crédito de ninguna entidad, solo al 20 \% le dieron un monto aproximado de 10,001-más, a diferencia de los resultados de Silva (2013), donde el $28.57 \%$ recibieron montos de créditos entre 3000 a 10000 nuevos soles y el 14.29 \% recibieron montos de créditos por más de 10000 nuevos soles. Esto demuestra que actualmente la gran mayoría de los representantes de las mypes no les han otorgado ningún monto, ya que estos no han accedido a la obtención de crédito en una entidad bancaria o no bancaria.

11. En el cuadro 11: El 80 \% al no solicitar crédito de ninguna entidad, solo el 20 \% utilizó el crédito obtenido para el mejoramiento y/o ampliación de su local, a diferencia de los resultados de Sagástegui (2010), donde el $100 \%$ de las mypes que recibieron crédito invirtieron dicho crédito en el mejoramiento y/o ampliación de sus locales. Esto demuestra que actualmente la gran mayoría de los representantes de las mypes no lo han utilizado, ya que estos no han accedido a la obtención de crédito en una entidad bancaria o no bancaria.

12. En el cuadro 12: El 80 \% no solicitó crédito de ninguna entidad; solo el $20 \%$ pagó una tasa de interés del 11-20\% por el crédito obtenido. Esto demuestra que actualmente la gran mayoría de los representantes de las mypes no pagaron ninguna tasa de interés, ya que estos no han accedido a la obtención de crédito en una entidad bancaria o no bancaria. 


\section{CONCLUSIONES}

\section{Referente a los representantes legales de las mypes}

- La totalidad de las mypes del sector servicio - rubro chifa de las ciudades de Santa y Coishco tiene edades entre los 26-60 años.

- La mayoría de los representes de las mypes del sector servicio - rubro chifa de las ciudades de Santa y Coishco son del sexo masculino, los cuales tienen estudios primarios y secundarios.

\section{Referente a las mypes}

- La mayoría de las mypes del sector servicio - rubro chifa de las ciudades de Santa y Coishco estableció la creación de su mype por una oportunidad en el mercado, los cuales tienen de 1,3 a más de 5 años de funcionamiento.

\section{Referente al financiamiento}

- La mayoría de las mypes del sector servicio - rubro chifa de las ciudades de Santa y Coishco tiene financiamiento propio, aunque se considera que las entidades bancarias otorgan mayores facilidades.

\section{REFERENCIAS BIBLIOGRÁFICAS}

Bustamante, C. (S/F). ¿Qué tipos de Financiamiento existen en el Mercado Peruano para las mypes? Recuperado el 22 de octubre del 2013, de: http://www.caballerobustamane. com.pe/plantilla/lab/ecb191009_2.pdf

Ferraros, C. (2009). Apoyando a las pymes: Políticas de fomento en América Latina y el Caribe. Recuperado el 21 de agosto del 2014, de: http://www.cepal.org/publicaciones/ xml/0/45410/lcr.2180.pdf

García, M. A. (2013). Pequeñas empresas. Recuperado el 22 octubre de 2013 de:_ http://www.nomeparo.eu/ayudas-a-emprendedores/pequenas-empresas n-31.htm.

Latínez, L. (2013). Financiamiento vía Banco de la Nación. Recuperado el 10 de noviembre de 2013 en: http://www.laprimeraperu.pe/online/economia/nueva-ley-demypes- financiamiento-via-banco-de-la-nacion_141430.html

Maudos, A. (2009). Eliminando barreras: El financiamiento a las pymes en América Latina. Recuperado el 21 agosto del 2014 de: http://www.cepal.org/ddpe/publicaciones/ xml/0/45220/Financiamiento_a_pymes.pdf

Tam, N. (2010). MYPES, motores del crecimiento. Recuperado el 30 octubre del 2013, de: http://laestrella.com.pa/online/impreso/2013/09/29/mypes-motores-del-crecimiento. asp. 